\title{
Método de registro gráfico de la forma externa de una momia
}

Eugenio Aspillaga ${ }^{1}$, Claudio Paredes ${ }^{1}$ Y MARIO GONZÁLEZ ${ }^{1}$

\section{Introducción}

Los estudios de paleopatología en restos humanos momificados conllevan muchas veces la destrucción parcial o total, durante la disección, de un material que por sus características es único, como lo son las momias humanas. Por lo cual es necesario disponer de muy buenos métodos de registro gráfico que den cuenta del aspecto general de la momia, antes de su disección, como un objeto tridimensional y la ubicación de los órganos dentro de ella, para complementar la descripción anatómica, las fotografías y otras formas de registro con objeto de poder "reconstruir" la momia, en sus aspectos principales, si fuese necesario.

Durante estudios de paleopatología, que involucraban la disección de aproximadamente 40 momias precolombinas, provenientes de la quebrada de Tarapacá, Segunda Región, Chile, en el ámbito de un proyecto de investigación dirigido por el profesor $\mathrm{J}$. Munizaga, fue necesario registrar lo más fielmente posible la forma, en su aspecto tridimensional, de troncos de momias y la ubicación relativa de sus órganos internos. Todo esto no era posible lograr tan sólo mediante fotografías y/o dibujos; esto nos llevó a desarrollar un método de registro gráfico, para complementar los trabajos de disección de momias, en especial de sus troncos, que no fuera demasiado complicado de aplicar y de escaso requerimiento material para su desarrollo.

\section{Descripción del método}

Para todos los efectos, en este trabajo los términos relativos a la orientación del cuerpo de la momia tales como: adelante, atrás, arriba, abajo, sagital, ventral, dorsal y otros, serán equivalentes a los

1 Departamento de Ciencias Sociológicas y Antropológicas de la Universidad de Chile, Santiago, CHILE. usados para las descripciones anatómicas normales del ser humano. Además consideraremos como punto de referencia externo al tronco de la momia, la horizontal establecida por la superficie de la mesa de disección.

La momia para todos los efectos de medición y descripción, asimismo, será ubicada decúbito dorsal sobre la mesa de trabajo, ubicando el tórax paralelo a la superficie de ésta.

Se establecieron los siguientes puntos, ejes, ángulos y planos y medidas de referencia, en el tronco de una momia:

1. Puntos establecidos en la norma frontal o ventral (Figura 1a).

- Punto anterior de la extremidad acromial en ambas clavículas, denominando Ai al punto de la clavícula izquierda y Ad al de la derecha.

- Centro de la parte anterior del manubrio del esternón: Punto B.

- Centro de la parte anterior de la apófisis xifoides: Punto D.

- Punto central en el esternón, entre los puntos B y D: Punto C.

- Punto más inferior del borde de ambas décimas costillas: Puntos Ed y Ei.

- Punto superior del borde de la cresta ilíaca, en ambos lados: Puntos Fd y Fi.

- Punto más anterior de la espina ilíaca anterosuperior, de ambos lados: Puntos Gd y Gi.

- Punto más superior de la parte anterior de la sínfisis púbica: Punto $\mathrm{H}$. 


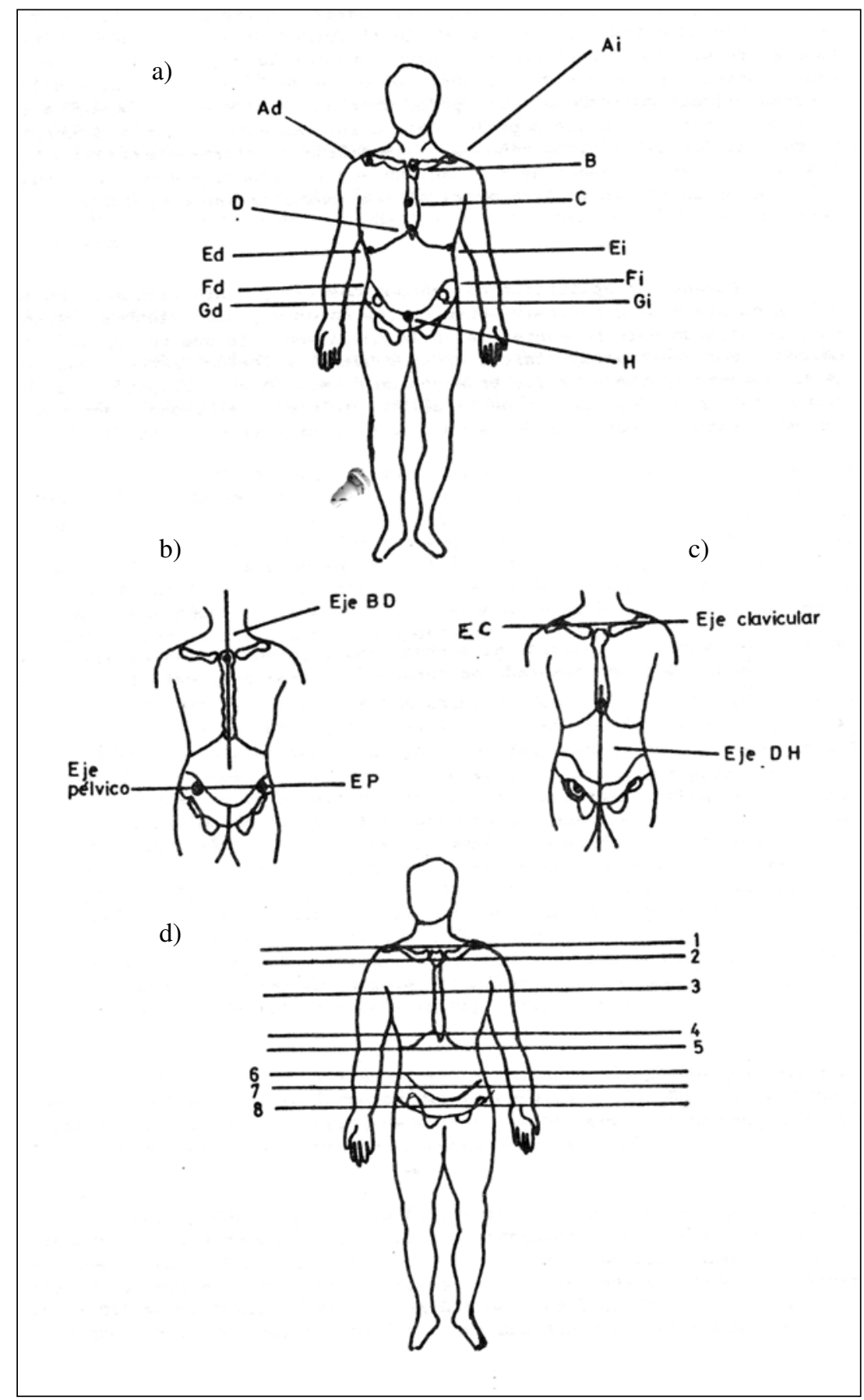

Figura 1. Puntos y ejes establecidos en la norma frontal o ventral.

2. Ejes establecidos para la norma frontal.

a) Se instituyeron dos ejes sagitales anteriores para el tronco de una momia humana. Un eje producto de la prolongación de la recta que une los puntos B esternón y D xifoides: Eje B D (Figura 1b) y otro formado por la recta que une los puntos D xifoides y $\mathrm{H}$ pubis: Eje D H (Figura 1c).

b) Se establecieron además dos ejes transversales anteriores: Un eje formado por la recta que pasa por los puntos Gd y Gi: Eje Pélvico EP. 
(Figura 1b) y un segundo eje formado por la recta que une los puntos Ad y Ai: Eje clavicular EC (Figura 1c).

3. Angulos de torsión de los ejes entre sí.

a) Angulo 1: Es el ángulo superior derecho, establecido en la intersección del eje BD con el eje clavicular EC.

b) Angulo 2: Está formado por la intersección de los ejes BD y DH, considerando el ángulo del lado hacia el cual se desvía el eje DH con respecto al eje BD.

c) Angulo 3: Es el ángulo superior derecho establecido en la intersección del eje DH y el eje pélvico, EP.

d) Angulo 4: Es el ángulo establecido entre el eje clavicular EG y el eje pélvico EP, utilizando como punto de rotación, para cada uno, los ejes sagitales correspondientes BD y DH respectivamente; midiéndose este ángulo con el observador localizado en el extremo inferior del tronco, mirando hacia la zona cefálica, y considerando el ángulo superior izquierdo (LI) si el eje pélvico se encuentra rotado en el sentido que giran las manecillas de un reloj, con respecto al eje clavicular EC considerándolo como horizontal, y el ángulo superior derecho (LD) si sucede lo contrario.

\section{Planos de corte.}

Se determinaron ocho planos transversales y uno sagital delimitados por el perímetro externo del tronco de una momia. Los diferentes planos son trazados en forma perpendicular a la superficie de la mesa de trabajo, así como también, con excepción del plano sagital, sus superficies y los ejes sagitales correspondientes. Cada uno de los planos se trazó a partir de los puntos señalados arriba.

a) Plano sagital PS, es el delimitado por la línea que sigue la forma externa del tronco de la momia y que une los puntos ubicados sobre los ejes sagitales BD y DH y descendiendo en torno al tronco de modo que el plano resultante sea perpendicular a la mesa de trabajo.

b) Primer plano transversal (Pl): Es el delimitado por una línea que define el contorno externo de la momia a nivel de las clavículas y que pasa sobre el eje clavicular EC (1 en Figura 1d).

c) Segundo plano transversal (P2): Está determinado por una línea que nace perpendicular al eje $\mathrm{BD}$ en el punto B y sigue rodeando la parte superior del tórax para terminar en el mismo punto por el otro lado del eje de modo que el plano resultante sea perpendicular a la mesa de trabajo.

d) Tercer plano transversal (P3): Formado de igual forma que el anterior $\mathrm{P} 2$ pero a partir del punto $\mathrm{C}$ y siguiendo por la parte media del tórax.

e) Cuarto plano transversal (P4): Formado de igual manera que los planos transversales $\mathrm{P} 2$ y $\mathrm{P} 3$ pero la línea parte del punto D.

f) Quinto plano transversal (P5): Está delimitado por la línea transversal que rodea la parte inferior del tórax de la momia, pasando por los puntos Ei y Ed.

g) Sexto plano transversal (P6): Es el delimitado por la línea que rodeando transversalmente el tronco pasa por los puntos Fd y Fi.

h) Séptimo plano transversal (P7) se forma igual que el plano anterior $\mathrm{P} 6$ pero pasando por los puntos Gd y Gi, es decir, por sobre el eje pélvico.

i) Octavo plano transversal (P8) igual que los anteriores pero pasando por el punto $\mathrm{H}$.

Todos estos planos transversales se indican en la Figura 1d.

5. Medidas entre puntos y planos.

a) Distancia de cada punto de los antes señalados con los adyacentes (p.e., distancia entre B y C, entre D y C.

b) Distancia entre Ad y Ai.

c) Distancia entre Ad y eje BD.

d) Distancia entre Gd y Gi.

e) Distancia entre Gd y eje DH.

f) Largo máximo del tronco. 
g) Distancia entre B y Ad y Ai.

h) Diámetro entre el eje sagital y la parte que se corresponde con la apófisis espinosa en el punto en que se localiza cada plano transversal.

Además de las medidas, planos, ángulos y ejes arriba señalados, se pueden establecer otros secundarios si así lo determinase el estado de conservación, torsión $\mathrm{u}$ otro que una momia en particular presente. En el caso de los planos se establecerán nuevos en forma intermedia a los ya establecidos.

\section{Aplicación del método}

\section{Materiales}

Se requieren los siguientes materiales para aplicar el método de registro tridimensional de la forma externa de una momia y la ubicación relativa de sus órganos internos:

- Huincha métrica metálica (2 m).

- Alambre de cobre de $4 \mathrm{~mm}$ de espesor (trozos de 50 y $150 \mathrm{~cm}$ ).

- Antropómetro.

- Papel milimetrado, un pliego de cartulina con cuadriculados de $5 \mathrm{~cm}$.

- Dos trozos de lienza de algodón, delgadas.

- Tiza de color, alfileres, transportador y dos reglas plásticas de $50 \mathrm{~cm}$ cada una.

\section{Procedimiento}

Una vez ubicada la momia decúbito dorsal sobre la mesa de disección, se procede a marcar con tiza los puntos definidos supra y a medir las distancias entre ellos y medir el largo total del tronco.

Para medir el ángulo dos se emplean dos lienzas, las cuales se trenzan haciéndolas coincidir con cada uno de los dos ejes sagitales y sobreponiendo el transportador a la intersección de las dos rectas resultantes. De igual manera se miden los ángulos 1 y 3 , superponiendo las lienzas a los ejes correspondientes. El ángulo 4 se mide poniendo el eje clavicular paralelo a la superficie de la mesa de trabajo y sobreponiendo una regla al eje pélvico, colocando una segunda regla horizontal para medir el ángulo resultante con el transportador.

Los diferentes planos son determinados por selchas o perfiles hechos con alambre de cobre que se amolda al contorno del tronco en la parte de este por la cual tenga que pasar un determinado plano. Dándose así al alambre la forma del tronco. En la sección por la que pasa un plano se marcan además sobre el alambre los puntos correspondientes al eje sagital y a la región de la apófisis espinosa que corta al plano delimitado, y se mide ese diámetro y el diámetro máximo obtenido. Posteriormente se retira la selcha ya moldeada, evitando deformarla y se la fija mediante alfileres en la cartulina cuadriculada, dejando hacia arriba el punto correspondiente al eje sagital y verticalmente hacia abajo el punto que se corresponde con la apófisis espinosa de esa sección, corrigiendo las distorsiones, producidas al retirar la selcha, mediante las medidas tomadas. A través de esto último es posible delimitar el plano sobre el que se aplicó la selcha y reducirlo a escala en papel milimetrado.

Una vez medidos los ángulos, delimitados los planos y dibujos en papel milimetrado, de la forma en que se muestra en la Figura 2 y consignadas todas las medidas arriba descritas, se procede a un examen externo de la momia, para posteriormente proceder a su disección.

Para poder referir cada órgano interno del tronco, es necesario proceder de la siguiente forma para su disección. En primer término se remueve la pared torácica, de uno de los lados, desarticulando las costillas de la columna vertebral a través de un corte que va desde la altura de la última vértebra cervical a la última dorsal en forma paralela y a unos tres $\mathrm{cm}$ del costado de ésta para proseguir por el borde inferior del tórax hacia adelante hasta los cartílagos esternales, los cuales se seccionan en forma paralela al esternón. Luego se desarticula la clavícula del manubrio del esternón para continuar con el corte hasta la columna. Todo esto permite retirar la pared torácica completa y así visualizar claramente la ubicación relativa de cada órgano con respecto al plano sagital visto del lado del cual se retiró la pared y a los planos transversales correspondientes. Se procede de igual forma con el otro lado. Así también se procede con el abdomen, para levantar la capa muscular, realizando cortes por ambos costados desde el corte de la base del tórax a las crestas ilíacas correspondientes para, unirlos, siguiendo el borde anterior del íleon y la parte superior del pubis. En este caso sólo se consigna la posición del hígado, riñones y otros órganos claramente delimitados. Los órganos por 
CLAVE: $C / T-14 \quad S-m^{\prime}$

Angulo $E C / E P=16^{\circ} \mathrm{Li}$
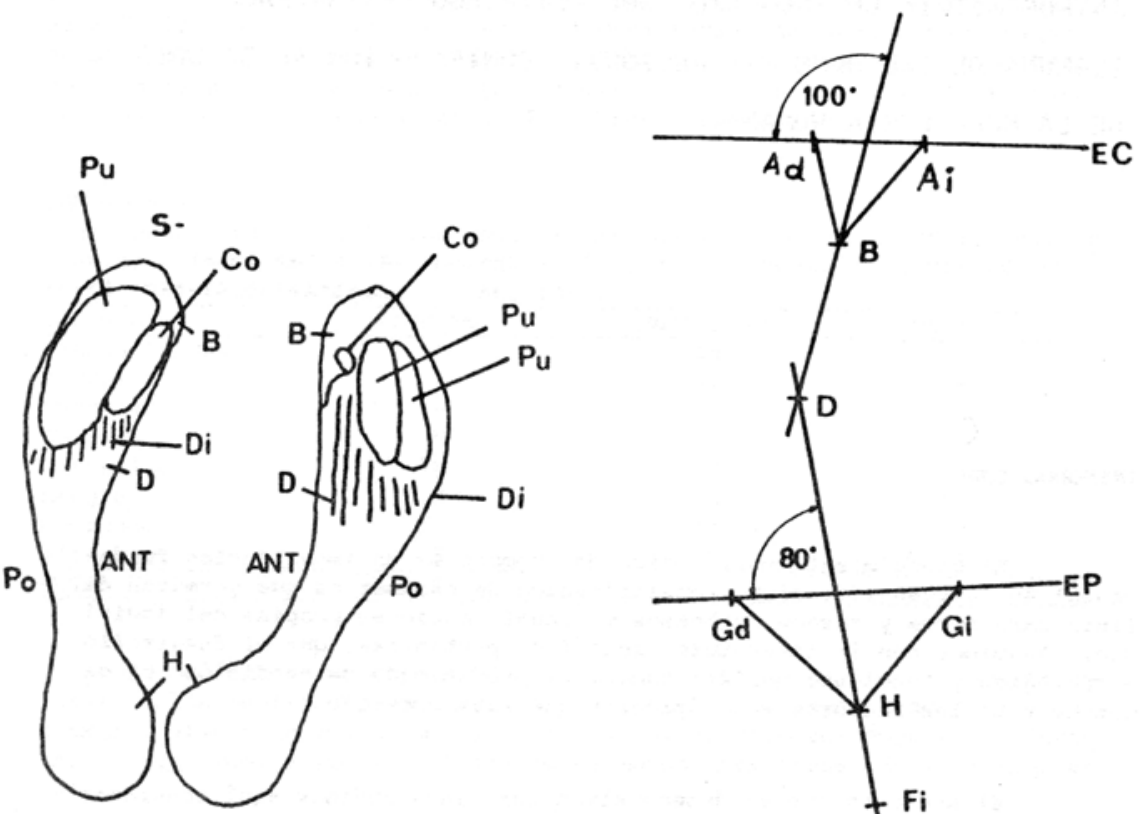

1.

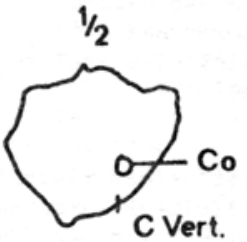

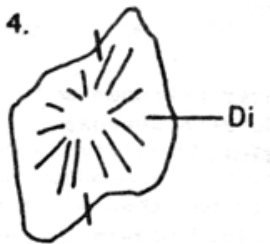

2.
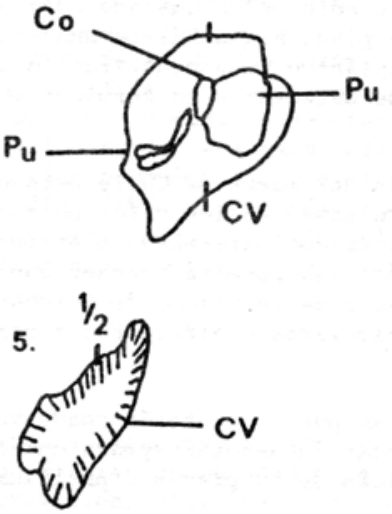

3.
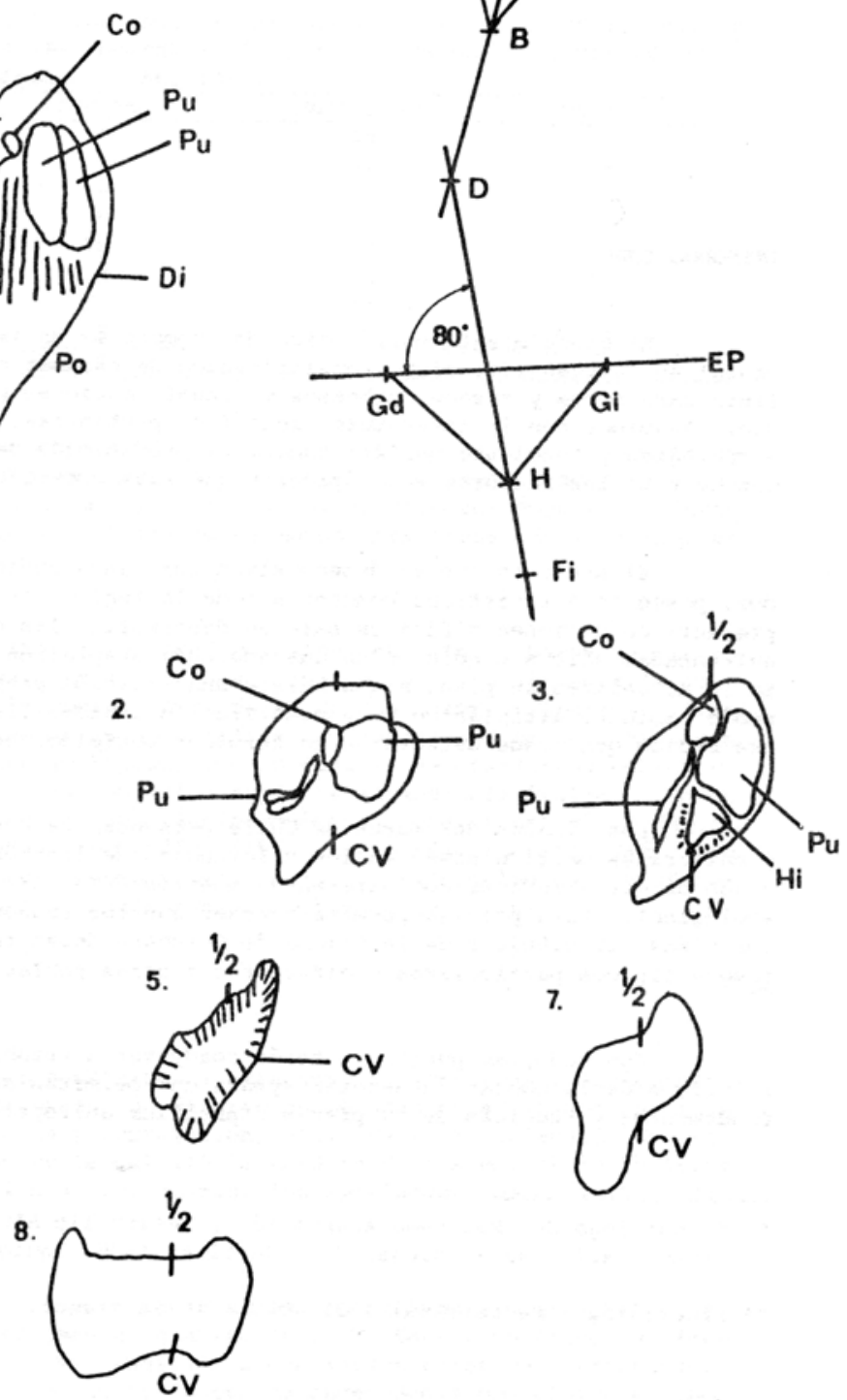

Figura 2. Medidas entre puntos y planos. 
su tamaño se dibujan, en sus secciones, a mano alzada auxiliándose de un compás y cinta métrica para conservar las proporciones relativas.

Durante la aplicación del método descrito, confeccionamos un modelo a escala basado exclusivamente en los datos y procedimientos expuestos, resultando éste bastante fiel a la momia sobre la que se aplicó el método. Por nuestra experiencia creemos que el referido método constituye un valioso aporte para los trabajos de disección y descripción de una momia contribuyendo, junto a un detallado protocolo de trabajo, a un registro más exacto de material de estudio de un enorme valor científico. ${ }^{2}$

2 La colección antropológica observada proviene de las excavaciones de L. Núñez en quebrada de Tarapacá (c/t-31 s-r; c/t-14 s-m; Tr-40/t-13). 